\title{
Atualização sobre a definição, consequências e diagnóstico da sarcopenia: uma revisão literária
}

Vitor Oliveira', Rafael Deminice ${ }^{2}$

\section{RESUMO}

Introdução: A sarcopenia é definida como uma desordem muscular esquelética progressiva e generalizada que está associada a maior probabilidade de complicações de saúde, incluindo quedas, fraturas, incapacidade física e mortalidade. Esta desordem, anteriormente entendida como uma condição inerente à idade, apresenta causas diferentes e afeta muitos indivíduos independentemente da idade. A sarcopenia é atualmente reconhecida como uma condição patológica e foi incluída na Classificação Internacional de Doenças (CID-10-CM - M62.84).

Objetivo: Apresentar uma revisão literária sobre as causas da sarcopenia, epidemiologia e forma e métodos de diagnóstico atualmente utilizados.

Fontes de dados: MEDLINE/PubMed, Embase e SciELO (pesquisa inicial) e referências bibliográficas dos artigos selecionados da pesquisa primária (pesquisa em cascata).

Métodos de revisão: Utilizou-se uma combinação dos seguintes termos: sarcopenia, diagnóstico, definição operacional, prevalência, consequências. Foram considerados artigos originais, artigos de revisão, diretrizes clínicas e consensos em inglês e português publicados desde 1989 (ano da proposta do termo sarcopenia) até maio de 2020.

Resultados: As principais referências selecionadas foram os artigos que tratavam da definição de sarcopenia. Foram também selecionados os artigos relacionados com testes e avaliações utilizados para o diagnóstico da sarcopenia e artigos que descrevem a prevalência da sarcopenia em diferentes populações.

Conclusões: Considerando a evolução histórica do diagnóstico da doença, é notório tentar aproximar o conhecimento científico da prática clínica com a sugestão de métodos e pontos de corte que facilitam a identificação imediata da sarcopenia. No entanto, há um elevado número de diferentes posições e definições operacionais de sarcopenia, o que dificulta a determinação da prevalência real. Apesar dos recentes desenvolvimentos na compreensão da sarcopenia, uma definição operacional definitiva e a melhor forma de diagnosticar a sarcopenia ainda são motivo de debate. Assim, acredita-se que a atual definição operacional não é definitiva e pode sofrer alterações consideráveis à medida que o conhecimento sobre a sarcopenia se desenvolve.

Palavras-chave: Sarcopenia; Fragilidade; Envelhecimento; Epidemiologia.

\section{INTRODUÇÃO}

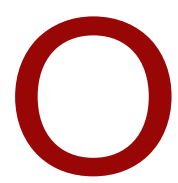

declínio das funções físicas e as mudanças na massa muscular que ocorrem com a idade têm sido estudados há muito tempo, mas o termo sarcopenia surgiu pela primeira vez na literatura em $1989,{ }^{1}$ numa tentativa de aumentar a atenção ao problema e obter reconhecimento das entidades de saúde da época. ${ }^{2}$ Desde então, a compreensão da doença evoluiu dramaticamente. Há vários anos que a sarcopenia é reconhecida como uma condição inerente à idade, mas hoje apresenta diferentes fatores de causalidade e afeta muitos indivíduos, independentemente da idade, gerando consequências para a saúde e aumento dos gastos com a saúde. Em

1. Department of Child, Family and Population Health Nursing, University of Washington Seattle. Washington, Unites States.

2. Departamento de Educação Física, Universidade Estadual de Londrina. Londrina, Paraná, Brasil. 
números absolutos estima-se que a sarcopenia afetava mais de 50 milhões de pessoas em todo o mundo em 2010, prevendo-se que atinja os 200 milhões até $2050 .^{3}$

Devido à sua importância e consequências, a sarcopenia foi reconhecida como uma condição patológica tendo sido incluída na Classificação Internacional de Doenças (CID-10-CM-M62.84), para além de existirem diferentes ferramentas e métodos de diagnóstico propostos para o diagnóstico desta patologia. ${ }^{4}$ Apesar dos progressos realizados relativamente à sarcopenia, não existe uma terapia farmacológica aprovada e todas as condutas utilizadas para melhorar esta condição são consideradas paliativas. ${ }^{5}$

Assim, tendo em conta a prevalência e a importância atual da sarcopenia para a saúde individual e coletiva, este artigo apresenta uma revisão de literatura sobre os recentes avanços no estudo da sarcopenia, incluindo a forma e os métodos de diagnóstico atualmente utilizados.

\section{MÉTODOS}

Procedeu-se a uma investigação bibliográfica não sistemática nas bases de dados eletrónicas MEDLINE/ /PubMed, Embase e SciELO (pesquisa inicial), utilizando uma combinação dos seguintes termos (termos livres e respetivos equivalentes MeSH em português e em inglês): sarcopenia, diagnóstico, definição operacional, prevalência, consequências. Foram considerados artigos originais, artigos de revisão, diretrizes clínicas e consensos em inglês e português publicados desde 1989 (ano da proposta do termo sarcopenia) até maio de 2020. Além disso, foi realizada uma investigação manual de referências bibliográficas dos artigos selecionados da pesquisa primária (pesquisa em cascata).

As principais referências selecionadas foram os artigos que tratam da definição de sarcopenia. Foram também selecionados os artigos relacionados com testes e avaliações utilizados para o diagnóstico da sarcopenia e artigos que descrevem a prevalência da sarcopenia em diferentes populações.

\section{RESULTADOS}

Definição conceitual, fatores causais e epidemiologia

O termo sarcopenia provém do grego, onde sarcosignifica carne/músculo e - penia significa deficiência. ${ }^{6}$ A sarcopenia é, atualmente, definida como uma desor- dem progressiva e generalizada do músculo esquelético, que está associada a uma maior probabilidade de complicações de saúde, incluindo quedas, fraturas, incapacidade física e mortalidade. ${ }^{?}$

Entre os fatores causais, o envelhecimento é reconhecido como a principal causa da sarcopenia. ${ }^{7}$ A massa muscular e a força variam ao longo da vida, diminuindo com o envelhecimento. Considera-se que a capacidade física máxima de um indivíduo é alcançada entre os 20 e os 30 anos de idade e que a redução desta capacidade surja de forma pronunciada após os 50 anos de idade. De acordo com a literatura, após esta idade há perdas anuais de 1 a $2 \%$ da massa muscular da perna e de 1,5 a $5 \%$ da força muscular. ${ }^{8}$

Ao longo dos anos tem-se observado que a sarcopenia não é uma condição que afeta apenas os idosos. Os indivíduos portadores de várias doenças crónicas específicas apresentam perda de força e de massa muscular de forma mais acentuada quando comparados com indivíduos sem estas doenças, independentemente da idade. Assim, para além do processo de envelhecimento natural, é amplamente reconhecido hoje em dia o papel que a presença de doenças inflamatórias crónicas desempenha no desenvolvimento da sarcopenia. ${ }^{3}$ Estudos recentes têm relatado perda excessiva de massa muscular, baixa força e fraco desempenho físico em pessoas com diferentes doenças crónicas, incluindo diabetes, insuficiência cardíaca, hipertensão, artrite, osteoporose, cancro, doença renal crónica, infeção do vírus da imunodeficiência humana, entre outras. ${ }^{9 \cdot 12}$ Atualmente, para além do envelhecimento, três fatores foram reconhecidos como potenciais causas da sarcopenia: ${ }^{7}$ a) doenças sistémicas, especialmente aquelas que aumentam os processos inflamatórios; $b$ ) inatividade física; e c) ingestão inadequada de energia ou proteína. Assim, considerando os seus fatores causais, a sarcopenia é atualmente classificada como: a) primária, relacionada com a idade, quando nenhuma condição que não o próprio envelhecimento esteja associada ao seu desenvolvimento; e b) secundária, quando há ocorrência de fatores de causalidade que não o envelhecimento (como doenças crónicas). ${ }^{3}$

Os estudos de prevalência da sarcopenia são mais robustos em indivíduos idosos, onde a prevalência estimada é de aproximadamente $10 \%$ da sarcopenia na população com mais de 60 anos de idade. ${ }^{13} \mathrm{Na}$ Europa, 
as projeções preveem números absolutos entre 18 e 32 milhões de indivíduos com sarcopenia com mais de 65 anos de idade até $2045 .{ }^{14}$ Espera-se que cerca de $200 \mathrm{mi}$ lhões de pessoas sejam afetadas pela sarcopenia até ao ano 2050 em todo o mundo. ${ }^{3}$ Além disso, diferentes estudos e revisões propuseram determinar a prevalência da sarcopenia nos idosos e em indivíduos com diferentes condições clínicas. A Tabela 1 apresenta uma síntese dos principais estudos publicados nos últimos dez anos com este objetivo.

\section{Sarcopenia aumenta custo dos cuidados de saúde e mortalidade}

A importância do estudo, diagnóstico e tratamento da sarcopenia deve-se às consequências atribuídas à sua presença. $O$ consenso atual sobre a sarcopenia caracteriza-a como uma desordem muscular esquelética associada a uma alta probabilidade de resultados adversos, incluindo quedas, fraturas, incapacidade física e mortalidade. ${ }^{7}$ Assim, procura-se nas definições teóricas da sarcopenia enfatizar as suas consequências a nível individual. Além das consequências físicas individuais, a sarcopenia é também um preditor da mortalidade por todas as causas, ${ }^{31-33}$ além de aumentar os custos de hospitalização. ${ }^{34}$

Regista-se uma crescente consciencialização sobre os custos dos tratamentos de saúde gerados pela sarcopenia, onde diferentes estudos têm reportado um aumento significativo dos custos de hospitalização quando esta condição está presente. ${ }^{34-36}$ Já em 2004, Janssen e colaboradores ${ }^{35}$ realizaram um estudo para estimar os custos dos tratamentos de saúde gerados pela sarcopenia, considerando que é uma condição que leva à incapacidade física e que idosos com sarcopenia precisariam de ajuda para realizar atividades básicas de vida diária, como o banho, vestir roupas, entre outros. Assim, estes autores estimaram que os custos diretos dos tratamentos de saúde atribuídos à sarcopenia nos Estados Unidos nos anos 2000 eram de USD 18,5 biliões, o que representou cerca de $1,5 \%$ do total das despesas de cuidados de saúde nesse ano. Além disso, estes autores estimaram uma despesa adicional de cuidados de saúde de USD 860 por cada homem e de USD 933 por cada mulher com sarcopenia. Estudos realizados na Europa também demonstraram a sarcopenia associada a elevados custos de hospitalização. Em Portugal,
Sousa e colaboradores ${ }^{34}$ demonstraram que a sarcopenia aumenta $58,5 \%$ os custos de hospitalização para doentes com idade $<65$ anos (mais $€ 1.240$ ) e $34 \%$ para os doentes com idade $\geq 65$ anos (mais $€ 721$ ). Na República Checa, Steffl e colaboradores ${ }^{36}$ demonstraram o dobro do custo do tratamento de saúde de idosos com sarcopenia em comparação com idosos sem sarcopenia (mais $€ 563,90$ ).

Outra preocupação trazida pela sarcopenia é a sua associação com a mortalidade por todas as causas, como demonstram diferentes estudos caracterizados pela sarcopenia primária e secundária. Liu e colaboradores $^{31}$ realizaram uma revisão sistemática, incluindo seis estudos, e demonstraram que o risco de mortalidade nos idosos com sarcopenia era maior do que nos idosos sem sarcopenia (risco relativo=1,60; IC95\% 1,24$-2,06)$. Villaseñor e colaboradores ${ }^{33}$ demonstraram que a sarcopenia está associada ao aumento do risco de mortalidade geral em sobreviventes de cancro da mama e pode também estar associada a uma mortalidade específica do cancro da mama (risco relativo=1,95; IC95\% $0,87-4,35)$. Hu e colaboradores ${ }^{32}$ demonstraram que os indivíduos com sarcopenia associada à desnutrição tinham quatro vezes mais probabilidades de morrer (risco relativo=4,78; IC95\% 2,09-10,97) em comparação com indivíduos sem sarcopenia e com nutrição normal. Assim, a sarcopenia é uma condição predominante em idosos com doenças crónicas, trazendo consequências tanto a nível individual como coletivo.

\section{Como determinar a sarcopenia? Considerações sobre a definição operacional}

Apesar dos recentes desenvolvimentos na compreensão da sarcopenia, uma definição operacional definitiva e a melhor forma de a diagnosticar são ainda motivos para o debate. ${ }^{37}$ No mais recente documento publicado em 2019 pelo Grupo de Trabalho Europeu sobre Sarcopenia em Pessoas Idosas, a sarcopenia é definida operacionalmente como baixa força muscular em associação com a baixa massa muscular. ${ }^{7}$ No entanto, foram propostas ao longo do tempo outras definições operacionais até que esta definição fosse alcançada. Esta revisão não pretende aprofundar as definições operacionais propostas antes de 2019, mas apresentar-se-á um breve resgate histórico para perceber como é que a sarcopenia chegou ao entendimento atual. 


\begin{tabular}{|c|c|c|c|c|c|}
\hline Referência & Local & Condição clínica & Amostra & Idade & Prevalência de sarcopenia \\
\hline Du et al. ${ }^{15}$ & Estados Unidos & $\begin{array}{l}\text { Aparentemente } \\
\text { saudável }\end{array}$ & $\begin{array}{l}4.367 \text { indivíduos, } \\
\text { incluindo } \\
2.458 \text { idosos } \\
\text { ( } \geq 65 \text { anos) }\end{array}$ & $\geq 18$ anos & $\begin{array}{l}\text { - } 18 \text { a } 39 \text { anos: } 4,25 \% \\
\text { - } 40 \text { a } 64 \text { anos: } 8,85 \% \\
\text { - } 65 \text { anos ou mais: } 15,51 \%\end{array}$ \\
\hline Diz et al. ${ }^{16}$ & $\begin{array}{l}\text { Revisão (só } \\
\text { estudos no } \\
\text { Brasil) } \\
\end{array}$ & $\begin{array}{l}\text { Aparentemente } \\
\text { saudável }\end{array}$ & $\begin{array}{l}31 \text { estudos e } \\
9.416 \text { indivíduos }\end{array}$ & $\geq 60$ anos & $17 \%$ (IC95\%, 13-22\%) \\
\hline Dodds et al. ${ }^{17}$ & Reino Unido & $\begin{array}{l}\text { Aparentemente } \\
\text { saudável }\end{array}$ & 719 indivíduos & $\begin{array}{l}\geq 85 \text { anos, } \\
\text { média de } \\
85,5 \text { anos }\end{array}$ & $21 \%$ \\
\hline Shafiee et al. ${ }^{13}$ & Revisão & $\begin{array}{l}\text { Aparentemente } \\
\text { saudável }\end{array}$ & $\begin{array}{l}35 \text { estudos e } \\
58.404 \text { indivíduos }\end{array}$ & $\geq 60$ anos & $\begin{array}{l}\text { - Homens: } 10 \% \text { (IC95\%, 8-12\%) } \\
\text { - Mulheres: } 10 \% \text { (IC95\%, 8-13\%) }\end{array}$ \\
\hline Yazar et al. ${ }^{18}$ & Turquia & $\begin{array}{l}\text { Aparentemente } \\
\text { saudável }\end{array}$ & 515 indivíduos & $\geq 18$ anos & $\begin{array}{l}\text { - } 18 \text { a } 39 \text { anos: } 0 \% \\
\text { - } 40 \text { a } 49 \text { anos: } 7 \% \\
\text { - } 50 \text { a } 59 \text { anos: } 10,6 \% \\
\text { - } 60 \text { a } 69 \text { anos: } 15,4 \% \\
\text { - } 70 \text { a } 79 \text { anos: } 21,2 \% \\
\text { - } 80 \text { anos ou mais: } 36,5 \%\end{array}$ \\
\hline Ngeuleu et al. ${ }^{19}$ & Marrocos & Artrite reumatoide & 123 indivíduos & $\begin{array}{l}\text { Média de } \\
52,3 \pm 13,2 \\
\text { anos }\end{array}$ & $39,8 \%$ \\
\hline Chindaprasirt $^{10}$ & Revisão & Cancro & - & - & $\begin{array}{l}\text { - Cancro do pulmão recebendo } \\
\text { quimioterapia paliativa: } 71 \% \\
\text { - Sobreviventes ao cancro de } \\
\text { mama: } 16 \% \\
\text { - Carcinoma hepatocelular } \\
\text { submetidos à hepatectomia } \\
\text { curativa: } 40,3 \% \\
\text { - Colangiocarcinoma submetidos } \\
\text { à hepatectomia com resseção } \\
\text { extra-hepática do ducto biliar: } \\
33 \%\end{array}$ \\
\hline Kim et al. ${ }^{20}$ & Revisão & Cirrose hepática & $\begin{array}{l}20 \text { estudos e } \\
4.037 \text { indivíduos }\end{array}$ & $\begin{array}{l}\text { Média de } \\
54,8 \text { anos } \\
(48,8-67,0)\end{array}$ & $\begin{array}{l}48,1 \% \text { (variando entre } 24,8 \text { e } \\
70 \% \text { nos estudos individuais) }\end{array}$ \\
\hline Murata et al. ${ }^{21}$ & Japão & Diabetes & 288 indivíduos & $\geq 65$ anos & $\begin{array}{l}\text { - Homens: } 15,2 \% \\
\text { - Mulheres: } 15,3 \%\end{array}$ \\
\hline \multirow[t]{2}{*}{ Wang et al. ${ }^{11}$} & China & Diabetes & 1.090 indivíduos & $\geq 60$ anos & $\begin{array}{l}14,8 \% \text { no grupo experimental vs } \\
11,2 \% \text { em grupo controlo } \\
\text { saudável }(p=0,035)\end{array}$ \\
\hline & & & & & (continua) \\
\hline
\end{tabular}




\begin{tabular}{|c|c|c|c|c|c|}
\hline Referência & Local & Condição clínica & Amostra & Idade & Prevalência de sarcopenia \\
\hline Kim et al. ${ }^{9}$ & Coreia do Sul & Diabetes & 810 indivíduos & $\begin{array}{l}\text { Média de } 58 \\
\text { anos }\end{array}$ & $\begin{array}{l}15,7 \% \text { no grupo experimental vs } \\
6,9 \% \text { em grupo controlo saudável } \\
(p<0,001)\end{array}$ \\
\hline Addison et al. ${ }^{22}$ & Estados Unidos & $\begin{array}{l}\text { Doença arterial } \\
\text { periférica }\end{array}$ & 108 indivíduos & $\begin{array}{l}>50 \text { anos, } \\
\text { média de } \\
68,7 \pm 0,6 \\
\text { anos }\end{array}$ & $\begin{array}{l}23,8 \% \text { no grupo experimental vs } \\
2,4 \% \text { em grupo controlo saudável } \\
(p<0,05)\end{array}$ \\
\hline Ryan et al. ${ }^{23}$ & Revisão & $\begin{array}{l}\text { Doença } \\
\text { inflamatória } \\
\text { intestinal }\end{array}$ & $\begin{array}{l}5 \text { estudos e } 658 \\
\text { indivíduos }\end{array}$ & $\begin{array}{l}\text { Mediana de } \\
33(32-39) \\
\text { anos }\end{array}$ & $41,6 \%$ \\
\hline Jones et al. ${ }^{24}$ & Reino Unido & $\begin{array}{l}\text { Doença pulmonar } \\
\text { obstrutiva crónica }\end{array}$ & 622 indivíduos & $\begin{array}{l}\text { Entre } 63 \text { e } \\
73 \text { anos }\end{array}$ & $14,5 \%$ (IC95\%, 11,8-17,4\%) \\
\hline Pereira et al. ${ }^{25}$ & Brasil & $\begin{array}{l}\text { Doença renal } \\
\text { crónica }\end{array}$ & 287 indivíduos & $\begin{array}{l}\text { Média de } \\
59,9 \pm 10,5 \\
\text { anos }\end{array}$ & $\begin{array}{l}5,9 \text { a } 9,8 \% \text {, dependendo do } \\
\text { método utilizado na avaliação da } \\
\text { massa muscular }\end{array}$ \\
\hline Souza et al. ${ }^{26}$ & Brasil & $\begin{array}{l}\text { Doença renal } \\
\text { crónica }\end{array}$ & 100 indivíduos & $\begin{array}{l}\text { Média de } \\
73,6 \pm 9,2 \\
\text { anos }\end{array}$ & $\begin{array}{l}11,9 \text { a } 28,7 \% \text {, dependendo da } \\
\text { definição operacional adotada }\end{array}$ \\
\hline Onishi et al. ${ }^{27}$ & Japão & Doenças digestivas & 303 indivíduos & $\begin{array}{l}\text { Mediana de } \\
70(28-92) \\
\text { anos }\end{array}$ & $32 \%$ \\
\hline Corallo et al. ${ }^{28}$ & Itália & Esclerose sistémica & 62 indivíduos & $\begin{array}{l}\text { Média de } 62 \\
\text { anos (32-78) }\end{array}$ & $42 \%$ \\
\hline Oliveira et al. ${ }^{12}$ & Revisão & HIV & $\begin{array}{l}13 \text { estudos e } \\
2.267 \text { indivíduos }\end{array}$ & $\begin{array}{l}\text { Média de } 35 \\
\text { a } 60 \text { anos } \\
\text { nos estudos } \\
\text { individuais }\end{array}$ & $24,1 \%$ (IC95\%, 17,8-31,0\%) \\
\hline Hao et al. ${ }^{29}$ & China & $\begin{array}{l}\text { Idosos } \\
\text { hospitalizados }\end{array}$ & 407 indivíduos & $\begin{array}{l}\text { Média de } \\
81 \pm 8 \text { anos }\end{array}$ & $31 \%$ \\
\hline Vetrano et al. ${ }^{30}$ & Itália & Parkinson & 210 indivíduos & $\begin{array}{l}\text { Média de } \\
73 \text { anos }\end{array}$ & $\begin{array}{l}\text { - Homens: } 45,6 \% \\
\text { - Mulheres: } 36,3 \%\end{array}$ \\
\hline
\end{tabular}

Nota: A comparação entre diferentes estudos de prevalência é frequentemente dificultada pela diferença de idades da amostra e também pela adoção de diferentes métodos, pontos de corte e definições operacionais de sarcopenia.

A Tabela 2 resume a história da definição operacional de sarcopenia nos últimos 30 anos. Inicialmente, a sarcopenia foi definida operacionalmente como baixa massa muscular. ${ }^{1}$ Assim, diferentes investigadores começaram a propor pontos de corte para o diagnóstico de sarcopenia com base na quantidade de massa mus- cular esquelética total e/ou apendicular. Os estudos de Baumgartner e colaboradores ${ }^{6}$ e de Janssen e colaboradores ${ }^{38}$ destacam-se neste item que, embora não tão recentemente, ainda hoje são usados como referência para o diagnóstico de baixa massa muscular. Baumgartner e colaboradores ${ }^{6}$ analisaram dados de um 
TABELA 2. Definições operacionais de sarcopenia propostas ao longo dos anos

\begin{tabular}{|c|c|c|}
\hline Ano & Definição operacional & Proponente \\
\hline 1989 & Baixa massa muscular & 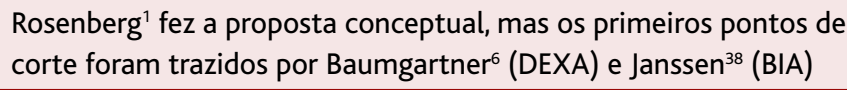 \\
\hline 2010 & $\begin{array}{l}\text { Baixa massa muscular em associação com baixa } \\
\text { função muscular }\end{array}$ & $\begin{array}{l}\text { Sociedade Europeia para Nutrição Clínica e Metabolismo, }{ }^{39} \text { Grupo } \\
\text { de Trabalho Europeu sobre Sarcopenia em Idosos }{ }^{3} \text { e Grupo de } \\
\text { Trabalho Internacional sobre Sarcopenia }{ }^{40}\end{array}$ \\
\hline 2019 & $\begin{array}{l}\text { Baixa força muscular em associação com baixa } \\
\text { massa muscular }\end{array}$ & Grupo de Trabalho Europeu sobre Sarcopenia em Idosos ${ }^{7}$ \\
\hline
\end{tabular}

Legenda: DEXA = Densitometria radiológica de dupla energia; $\mathrm{BIA}=$ Bioimpedância elétrica.

estudo baseado na população (The New Mexico Elder Health Survey, 1993-1995) realizado no Novo México, Estados Unidos, para desenvolver um método que estime a prevalência da sarcopenia. Foram analisados dados de 883 homens e mulheres idosos brancos, hispânicos e não-hispânicos. Utilizando a densitometria radiológica de dupla energia (DEXA), estes autores definiram a sarcopenia como um índice de massa muscular apendicular (IMMA, massa muscular apendicular dividida por quadrado de altura) abaixo de dois desvios-padrão de uma população de referência. Janssen e colaboradores $^{38}$ analisaram dados de 4.449 idosos ( $\geq 60$ anos), que participaram no Terceiro Inquérito Nacional de Saúde e Nutrição (Third National Health and Nutrition Examination Survey, NHANES), nos Estados Unidos, durante o período de 1988 a 1994, com o objetivo de determinar pontos de corte da quantidade de músculo esquelético que estavam associados ao risco de desenvolver incapacidade física. Neste estudo foram incluídos indivíduos brancos e negros não-hispânicos, bem como indivíduos da etnia mexicana/americana. A incapacidade física foi avaliada através de um questionário e o índice de massa muscular total (IMM, massa muscular total dividida por quadrado de altura) foi estimado usando bio impedância elétrica (BIA). Assim, é possível observar, mesmo na literatura recente, que os pontos de corte estabelecidos por Baumgartner e colaboradores $^{6}$ são os mais utilizados quando a massa muscular é estimada através do DEXA, enquanto os pontos de corte estabelecidos por Janssen e colaboradores ${ }^{38}$ são os mais utilizados quando a massa muscular é estimada por BIA.
Ao longo dos anos, e a partir das novas evidências científicas, tem havido um movimento mundial proposto por diferentes investigadores para formar grupos de trabalho e investigação sobre sarcopenia: a) o Grupo Especial de Trabalho sobre a caquexia-anorexia em doenças crónicas que causam a perda de peso (Special Interest Group on Cachexia-Anorexia in Chronic Wasting Diseases) e o Grupo Especial de Trabalho sobre nutrição geriátrica (Special Interest Group Nutrition in Geriatrics), que publicaram em 2010 uma posição oficial da Sociedade Europeia de Nutrição Clínica e Metabolismo sobre sarcopenia e caquexia; ${ }^{39}$ b) o Grupo de Trabalho Europeu sobre a sarcopenia em idosos (European Working Group on Sarcopenia in Older People, que será referido no presente estudo como EWGSOP1), que publicou em 2010 um consenso europeu sobre a definição e o diagnóstico da sarcopenia; ${ }^{3}$ e c) o Grupo de Trabalho Internacional sobre a Sarcopenia, que publicou em 2011 um consenso sobre a definição da doença. ${ }^{40}$ Os grupos de estudo e investigação aqui mencionados tinham um ponto comum, onde a importância da avaliação da função muscular (força muscular e/ou função física) foi reconhecida, juntamente com a massa muscular para o diagnóstico da sarcopenia. ${ }^{3}$ Assim, sugeriu-se que a sarcopenia fosse definida operacionalmente como baixa massa muscular em associação com a baixa função muscular. Entre estes grupos destaca-se o trabalho do EWGSOP1, porque, desde a sua publicação em 2010, a sua definição operacional tornou-se a mais usada nos estudos de sarcopenia.

Além de proporem alterar a definição operacional de sarcopenia, o trabalho destes grupos foi de grande 
relevância porque sistematizaram o diagnóstico desta doença, aumentando assim a consciencialização e a compreensão sobre o assunto. Esta sensibilização e compreensão também promoveram um aumento do número de pessoas interessadas na investigação da sarcopenia (registou-se um aumento de 8,1 vezes no número de artigos publicados na base de dados PubMed de 2010 a 2019). Como resultado, muitos aspetos que ligam as condições musculares esqueléticas patológicas a resultados desfavoráveis relacionados com a saúde foram identificados e são hoje mais bem compreendidos. Existem evidências científicas recentes que demonstram que a redução da força muscular é um melhor preditor dos resultados adversos relacionados com o sistema neuromuscular (e.g., quedas nos idosos, fraturas, incapacidade física, risco de hospitalização e mortalidade) do que a redução da quantidade de massa muscular. ${ }^{41-43}$ Por exemplo, Leong e colaboradores ${ }^{42}$ analisaram dados de 139.691 indivíduos incluídos no estudo Prospective Urban Rural Epidemiology Study e descobriram que a força do aperto de mão (também conhecida como força de preensão manual) está inversamente associada à mortalidade por todas as causas; a força do aperto de mão foi também um melhor preditor da mortalidade por doença cardiovascular e mortalidade por todas as causas do que a pressão arterial sistólica. Além disso, para comparar medidas de força muscular e massa muscular, Menant e colaboradores ${ }^{43}$ investigaram se diferentes definições operacionais de sarcopenia eram capazes de prever resultados funcionais e de saúde em 419 indivíduos idosos (idade média de $81,2 \pm 4,5$ anos, sendo $49 \%$ mulheres); estes autores concluíram que uma simples avaliação da força dos membros inferiores era tão eficaz para prever o equilíbrio, a mobilidade funcional e as quedas nos idosos como medidas mais caras e morosas da massa muscular. Da mesma forma, Schaap e colaboradores ${ }^{41}$ descobriram que a baixa força muscular está associada ao declínio funcional e este resultado não foi observado para a baixa massa muscular.

Considerando estas novas evidências, o EWGSOP1 reuniu-se novamente e publicou em 2019 uma atualização do consenso europeu sobre a definição e diagnóstico da sarcopenia (referido no presente estudo como EWGSOP2). O EWGSOP2 destaca-se por incluir a baixa força muscular como o resultado primário da sarcopenia (em vez de baixa massa muscular), ${ }^{7}$ gerando uma mudança significativa na compreensão e diagnóstico dessa doença, tanto na prática clínica como no campo científico. Assim, a sarcopenia começou a ser definida operacionalmente como baixa força muscular em associação com a baixa massa muscular.

\section{Diagnóstico}

Após a apresentação de conceitos, definições e contextualização da sarcopenia, discutir-se-á em pormenor o protocolo para o diagnóstico proposto pelo EWGSOP2. Esta proposta foi escolhida porque é a mais atual e, principalmente, porque incorpora toda a evolução dos conhecimentos adquiridos pela área ao longo dos anos. De acordo com o EWGSOP2, o indivíduo pode ser classificado numa das quatro categorias, tendo em conta a presença e gravidade da sarcopenia. A categorização baseia-se no uso de pontos de corte para força, massa muscular e função muscular apresentada na Tabela 3. Assim, os indivíduos avaliados podem ser classificados como: a) sem sarcopenia, quando a força muscular está acima do ponto de corte estabelecido; b) sarcopenia provável, quando a força muscular está abaixo, mas a massa muscular está acima do ponto de corte estabelecido; c) sarcopenia, quando a força e a massa musculares estiverem abaixo do ponto de corte; e d) sarcopenia severa, quando além da força e massa musculares, o indivíduo ainda apresenta a função física abaixo do ponto de corte estabelecido. Para a determinação da baixa força, massa muscular e função física, o EWGSOP2 apresenta ainda a possibilidade de diferentes testes e pontos de corte (Tabela 3 ).

O EWGSOP2 propõe ainda que seja seguido um algoritmo no diagnóstico da sarcopenia, com base no que os autores chamam de «Observação-Avaliação-Confirmação-Gravidade» (Find-Assess-Confirm-Severity). ${ }^{7} \mathrm{O}$ algoritmo sugere que o indivíduo seja encaminhado para avaliação clínica se se suspeitar de sarcopenia. Para determinar esta suspeita inicial, o EWGSOP2 propõe a aplicação do SARC-F, um questionário com itens que refletem alterações no estado de saúde associados às consequências da sarcopenia. ${ }^{44}$ No entanto, um estudo para validar a versão do questionário SARC-F em português não obteve resultados satisfatórios como ferramenta de triagem para a sarcopenia. ${ }^{45}$ Assim, na rotina clínica, sugere-se que o examinador 
dispense a aplicação do SARC-F e use um dos testes de força muscular apresentados na Tabela 3 para o diagnóstico inicial de sarcopenia. Quando não for possível, a aplicação e interpretação dos resultados do SARC-F deve ser adicionada aos conhecimentos e experiência do examinador, tendo o cuidado de evitar resultados falsos negativos.

A partir da aplicação do teste de força, se for detetada baixa força muscular, o indivíduo é diagnosticado como «sarcopenia provável» e a quantidade ou qualidade da massa muscular deve ser avaliada. Se for detetada baixa massa muscular, o indivíduo é diagnosticado como «sarcopenia» e a sua função física deve ser testada. Finalmente, se for detetada baixa função física, o indivíduo é diagnosticado como «sarcopenia grave».

\section{Componentes da definição operacional de sarcopenia}

Como anteriormente apresentado, o diagnóstico de sarcopenia envolve a avaliação de três parâmetros: a) força muscular; b) massa muscular; e c) função física. Relativamente a estes parâmetros existem atualmente diferentes ferramentas e métodos de diagnóstico propostos, alguns dos quais já estão estabelecidos e outros ainda em fase experimental. ${ }^{4} \mathrm{O}$ EWGSOP2 também divide os métodos entre os aplicáveis na prática clínica (baixo custo e aplicação fácil) e os que devem ser utilizados em estudos de investigação (maior especificidade e custo mais elevado). ${ }^{7}$ Embora existam métodos experimentais e alternativos muito promissores, estes são frequentemente de elevado custo ou ainda não há nenhuma proposta de pontos de corte ou valores de comparação a utilizar. Considera-se preferível a utilização de métodos já estabelecidos, uma vez que existe uma maior sistematização destes métodos, além de facilitar a comparação entre diferentes estudos e avaliações. Os métodos e procedimentos mais utilizados para deter- minar a força muscular, a massa muscular e a função física serão detalhados seguidamente.

\section{Força muscular}

A força muscular é o primeiro parâmetro a ser avaliado no diagnóstico da sarcopenia de acordo com o EWGSOP2. A força do aperto de mão é a forma mais habitual e simples de avaliar a força muscular, especialmente na população e no contexto clínico. ${ }^{42}$ Além da força muscular total, a força do aperto de mão diminui ao longo da idade adulta, apresentando uma boa validade preditiva, onde baixos níveis de força do aperto de mão estão associados a um maior número de quedas, incapacidade física, agravamento da qualidade de vida, maior duração da hospitalização e mortalidade.$^{46} \mathrm{~A}$ força do aperto de mão é medida com um dinamómetro de aderência manual e os principais tipos de dinamómetro são hidráulicos, pneumáticos ou mecânicos. Entre os diferentes tipos e marcas de dinamómetro disponíveis, o dinamómetro hidráulico Jamar® (JLW Instruments, Chicago, EUA) é o mais utilizado na literatura, além de ser comummente usado como padrão-ouro para validação de outros dinamómetros. ${ }^{46}$ 
Embora muitos estudos não relatem os procedimentos específicos adotados para a medição da força do aperto de mão, ${ }^{46-47}$ existem atualmente dois protocolos estabelecidos para este teste: o protocolo da Sociedade Americana de Terapeutas de Mão, atualizado em $2015,{ }^{48}$ e o protocolo Southampton, publicado em $2011 .{ }^{46} \mathrm{O}$ protocolo da Sociedade Americana de Terapeutas de Mão apresenta mais detalhes sobre a medição, o que pode facilitar a normalização. É importante que os estudos reportem explicitamente o protocolo adotado durante a medição da força do aperto de mão e que as alterações nos protocolos sem razão sejam fortemente desencorajadas. Os detalhes específicos dos protocolos são descritos na Tabela 4 .

Além do já estabelecido teste de aderência manual, o EWGSOP2 sugere como alternativa o uso do teste de sentar-levantar cinco vezes como medida de força muscular. Este teste só deve ser utilizado quando a força do aperto de mão não puder ser medida (e.g., em casos de artrite avançada) e nunca deve ser utilizado como a primeira opção de teste. ${ }^{7} \mathrm{O}$ teste de sentar-levantar consiste em levantar-se completamente da cadeira, o mais rápido possível, cinco vezes seguidas e sem parar entre repetições. Para efetuar o teste é utilizada uma cadeira com encosto reto, sem apoio para os braços, com aproximadamente 45 centímetros de altura, que deve estar encostada à parede ou estabilizada de alguma forma para evitar que se mova durante o teste. $O$ teste segue os seguintes passos: ${ }^{49}$

1. Inicialmente é realizado um pré-teste, onde o indivíduo será solicitado a levantar-se da cadeira apenas uma vez. Demonstre e explique os procedimentos ao indivíduo. Primeiro, cruze os braços sobre o peito e sente-se com os pés no chão. Então, levante-se completamente, mantendo os braços cruzados sobre o peito e sem tirar os pés do chão.

2. Após a demonstração certifique-se de que o indivíduo entendeu como o teste é realizado. Certifique-se também de que o indivíduo está sentado, ocupando a maior parte do assento, mas com os pés bem apoiados no chão. Peça ao indivíduo para se levantar completamente uma vez, mantendo os braços cruzados sobre o peito.

3. Se o indivíduo for capaz de realizar o pré-teste, proceda à avaliação. Instrua o indivíduo a levantar-se por completo o mais rápido possível cinco vezes se- guidas, sem parar entre repetições e mantendo sempre os braços cruzados sobre o peito. Quando o indivíduo estiver bem sentado e pronto para começar, avise-o que vai iniciar o temporizador, dizendo: "Prepare-se, agora!" Conte em voz alta cada vez que o indivíduo se levanta até à quinta vez. Pare o temporizador quando o paciente levantar completamente pela quinta vez, desta vez sendo o resultado final do teste. $\mathrm{O}$ teste deve ser interrompido antes do fim se o paciente utilizar os braços ou quando o avaliador considerar necessário para a segurança do indivíduo.

Em geral, o teste de sentar-levantar é pouco investigado pela sua validade preditiva. Estudos recentes demonstraram uma diferença significativa na prevalência da sarcopenia observada ao comparar os resultados obtidos com a força do aperto de mão com os resultados do teste de sentar-levantar cinco vezes. ${ }^{50}$

\section{Massa muscular}

Para o diagnóstico de sarcopenia, a massa muscular pode ser avaliada pela sua quantidade (massa muscular absoluta em kg) ou qualidade (massa muscular relativa à altura em $\mathrm{kg} / \mathrm{m}^{2}$ ) e pode ser estimada por diferentes métodos. Como primeira opção sugere-se a utilização do DEXA, o método padrão-ouro para a determinação da densidade mineral óssea e que tem sido amplamente utilizado para estimar a quantidade de massa muscular. O DEXA é um método fácil de aplicar onde não é necessária preparação do paciente. No entanto, este método apresenta limitações, onde o peso e o tamanho elevados do avaliado podem diminuir a precisão dos valores de massa muscular. ${ }^{51}$ Além disso, o custo do exame é relativamente elevado quando comparado com outros métodos (como a BIA) e pode ser um impedimento. Com os dados do DEXA, o examinador terá disponível a quantidade de massa muscular apendicular (massa muscular dos braços e pernas). A partir destes valores, a qualidade muscular é calculada, um indicador também conhecido como Índice de Massa Muscular Apendicular. O seu cálculo é realizado dividindo a quantidade de massa muscular apendicular pelo quadrado de altura em metros e expressa-se em $\mathrm{kg} / \mathrm{m}^{2}$.

Alternativamente, a BIA pode ser usada para estimar a massa muscular. A BIA transmite uma corrente 
TABELA 4. Protocolos para a mensuração da força do aperto de mão (preensão manual)

Sociedade Americana de Terapeutas de Mão

- Posicionamento do indivíduo. Sentado confortavelmente numa cadeira sem apoios para os braços, com os pés totalmente apoiados no chão, os quadris o mais afastados possível na cadeira e os quadris e joelhos posicionados a aproximadamente 90 graus.

- Posição do braço e do pulso. O braço a avaliar deve ter o ombro aduzido, o cotovelo flexionado a 90 graus e o antebraço e o pulso numa posição neutra. $O$ pulso deve ser posicionado entre 0 e 30 graus de extensão (dorsifexão) e entre 0 e 15 graus de desvio ulnar. Para evitar padrões de substituição muscular e garantir a adução do ombro recomenda-se que o indivíduo mantenha um pequeno bloco entre o braço avaliado e o tórax lateral. Durante o teste, o indivíduo deve ser lembrado para manter a sua posição e deve ser corrigido conforme necessário.

- Posicionamento do dinamómetro. Deve utilizar-se a segunda posição da alavanca do dinamómetro. O dinamómetro deve ser colocado na mão do indivíduo pelo examinador, que deve apoiar suavemente a base do instrumento para evitar quedas acidentais.

- Procedimentos para o teste. A força do aperto de mão deve ser aplicada suavemente, sem movimentos rápidos de puxar ou empurrar. Não deve ser fornecido qualquer feedback visual ou auditivo; por conseguinte, a marcação do dinamómetro deve ser afastada dos indivíduos, de modo que não possam ver o valor.

- Instruções. Como as instruções influenciam o desempenho nos testes de avaliação foram utilizadas instruções padronizadas em estudos de investigação que examinam a fiabilidade e a validade dos testes de força do aperto de mão. Para garantir a consistência, os indivíduos não devem ser treinados ou encorajados durante o teste e apenas devem ser fornecidas instruções verbais padronizadas. As instruções sugeridas incluem: "Este teste indicará a sua máxima força do aperto de mão. Quando eu disser 'vai', pressione o mais forte que puder até eu dizer 'pare'. Antes de cada tentativa vou perguntar 'Você está pronto?' e depois direi 'Vai'. Pare imediatamente se sentir alguma dor ou desconforto incomum em qualquer momento do teste. Você tem alguma pergunta? Você está pronto? Vai!" Então, quando o indivíduo começa a apertar o dinamómetro, o examinador deve dizer: "Mais forte ... mais forte ... mais forte ... relaxe". O examinador deve pedir ao indivíduo que relaxe quando o mostrador do dinamómetro estiver nivelado e começar a cair, após aproximadamente 3 a 5 segundos de aperto de mão.

- Tentativas e valores. Três tentativas com cada mão, alternando os lados, com pelo menos 15 segundos de descanso entre cada tentativa. A média de cada mão é usada.

- Equipamento a ser utilizado. Dinamómetro hidráulico manual JAMAR ou JAMAR Plus+.
Southampton

- Posicionamento do indivíduo. Sentado confortavelmente numa cadeira padrão com pernas, apoio para as costas e apoio fixo para braços. Pés apoiados no chão. Utilize a mesma cadeira para todas as medições.

- Posição do braço e do pulso. Os antebraços devem ser apoiados nos braços da cadeira, com o pulso logo após a extremidade do braço da cadeira (pulso em posição neutra, polegar voltado para cima).

- Posicionamento do dinamómetro. Posicione a mão de modo que o polegar fique em torno de um dos lados da pega e os quatro dedos estejam ao redor do outro lado. O dinamómetro deve ficar confortável na mão. Se necessário, mude a posição da pega. $O$ avaliador deve apoiar a base do dinamómetro na palma da mão enquanto o indivíduo segura o dinamómetro. O objetivo é suportar o peso do dinamómetro (para remover o efeito da gravidade na força máxima), mas deve-se ter cuidado para não restringir o movimento.

- Procedimentos para o teste. Demonstre como utilizar o dinamómetro para mostrar que ao apertar demasiado obtém uma pontuação melhor Comece com a mão direita. Encoraje o indivíduo a apertar o máximo de tempo e o mais forte possível ou até que o marcador pare de subir. Quando o marcador parar de subir, o indivíduo pode ser instruído a parar de apertar. Leia a força do aperto de mão em quilogramas no visor externo e grave o resultado no valor mais próximo de $1 \mathrm{~kg}$ no formulário de recolha de dados. Repita a medição na mão esquerda. Efetuar duas medições adicionais para cada mão, alternando os lados até obter três leituras no total para cada mão.

- Instruções. "Quero que aperte o mais forte que puder e o máximo de tempo possível, até que eu diga 'pare'. Aperte, aperte, aperte, pare" (quando o marcador parar de subir).

- Tentativas e valores. Três tentativas com cada mão, alternando os lados (começando pela mão direita). A melhor das seis medidas é utilizada.

- Equipamento a ser utilizado: Dinamómetro hidráulico manual JAMAR modelo J00105. 
elétrica indolor no corpo do paciente através de elétrodos posicionados na mão e no pé, determinando a resistência do corpo a esta corrente elétrica. A BIA é amplamente utilizada para a determinação da água total do corpo. É possível encontrar na literatura vários estudos validando equações para a estimativa da massa muscular a partir dos resultados da BIA. Neste sentido, sugere-se o uso da equação proposta por Kyle e colaboradores, ${ }^{52}$ que foi validada numa população diversificada e permite a previsão da massa muscular apendicular em indivíduos saudáveis e pacientes entre 22 e 94 anos de idade. A equação é a seguinte:

Massa muscular apendicular $=-4,221+(0,267 \mathrm{x}$ altura $^{2} /$ resistência $)+(0,095 \times$ massa corporal $)+(1,909$ $\mathrm{x}$ sexo $)+(-0,012 \mathrm{x}$ idade $)+(0,058 \mathrm{x}$ reatância $)$

[Onde: têm-se massa muscular apendicular em quilos, altura em centímetros, resistência em ohms, massa corporal em quilos, sexo sendo 1 para homens e 0 para mulheres, e reatância em ohms; $r=0,976$; erro padrão de estimativa de 1,12 quilos.]

Para obter resultados fiáveis da BIA é muito importante utilizar um aparelho tetrapolar e adotar um protocolo de medição, que inclua: ${ }^{33}$ a) medir pelo menos duas horas após a última refeição, e 30 minutos após a urina; b) o indivíduo não deve usar objetos metálicos (e.g., colar, pulseira, relógio, brincos, óculos, anel, jeans, entre outros), que devem ser removidos antes do teste; (c) posicionar o indivíduo vestido, descalço e sem meias, deitado numa posição supina numa maca, com os braços ligeiramente separados do corpo e as pernas confortavelmente separadas; d) assepsia anterior da pele com álcool $70 \%$ e posicionamento correto dos quatro elétrodos no lado direito do corpo, na mão (superfície proximal à falange do metacarpo), punho (na linha média entre a proeminência final do rádio e ulna), pé (superfície proximal à falange do metatarso) e tornozelo (na linha média entre os maléolos medial e lateral do tornozelo).

Além do DEXA e da BIA, a massa muscular pode ser avaliada por ressonância magnética ou tomografia computorizada, que são considerados os principais métodos para a avaliação não invasiva da massa muscular. Estes métodos têm um elevado custo financeiro e geralmente estão disponíveis apenas em grandes centros de investigação. A sua utilização é também limitada pela necessidade de profissionais altamente treina- dos e pela falta de pontos de corte para a baixa massa muscular. ${ }^{7}$ Embora difundida, a antropometria como ferramenta para determinar a massa muscular não é indicada devido ao elevado erro técnico associado a esta medida.

\section{Função física}

O método mais completo sugerido para a avaliação da função física é a Bateria Curta de Desempenho Físico (do inglês, Short Physical Performance Battery, SPPB), uma bateria que é fácil de aplicar e compreende três testes: ${ }^{54}$ a) equilíbrio estático, que envolve posicionar os pés em três posições diferentes, que devem ser mantidas até 10 segundos; b) velocidade de marcha, que consiste em caminhar uma distância de quatro metros, duas vezes, e o tempo mais rápido é utilizado como resultado; c) sentar e levantar da cadeira, conforme descrito na sessão sobre a força muscular. Após o desempenho, os testes são pontuados tendo em conta o tempo que o indivíduo teve na realização de cada um, resultando na pontuação final da SPPB que pode variar entre zero e doze pontos. ${ }^{54}$

Para realizar o teste, o examinador deve seguir um protocolo previamente determinado, com instruções específicas. O protocolo detalhado pode ser encontrado nas páginas 149 a 155 da dissertação de mestrado de Márcia Nakano (disponível no endereço eletrónico http://repositorio.unicamp.br/handle/REPOSIP/ 252485), juntamente com a forma de pontuação da SPPB. Nakano e colaboradores ${ }^{49}$ realizaram a adaptação cultural e avaliação da fiabilidade da SPPB para a população brasileira, disponibilizando um roteiro na língua portuguesa.

\section{CONCLUSÕES}

A sarcopenia tem grande relevância para a saúde, tanto do ponto de vista individual como populacional. Esta condição tem sido associada recentemente ao aumento da morbimortalidade nos idosos e pessoas com várias doenças crónicas, além de aumentar o custo do tratamento destas doenças. Esta relevância é evidente com o reconhecimento da sarcopenia como condição patológica em $2016 .{ }^{4}$ Assim, a compreensão e melhoria das formas e métodos de diagnóstico é particularmente significativa, uma vez que a sarcopenia não tem cura ou tratamento farmacológico aprovado. Assim, todas as 
condutas de tratamento de sarcopenia utilizadas hoje são consideradas paliativas e/ou experimentais. ${ }^{5}$

Considerando a evolução histórica do diagnóstico da doença, é notório tentar aproximar o conhecimento científico da prática clínica com a sugestão de métodos e pontos de corte que facilitam a identificação imediata da sarcopenia. No entanto, o elevado número de diferentes posições e definições operacionais dificulta a determinação da prevalência real e das condições associadas à sarcopenia em diferentes populações. Estudos demonstraram que a prevalência de sarcopenia é significativamente diferente quando a mesma população é testada usando diferentes definições operacionais. . $^{50,55}$

Apesar da iniciativa de incorporar medidas de função muscular (força e desempenho físico) no diagnóstico da sarcopenia há críticas a esta proposta. Dawson-Hughes e Bischoff-Ferrari ${ }^{37}$ demonstraram que o IMMA por si só é um preditor significativo de quedas e o mesmo resultado não foi encontrado para o IMMA associado à força de aperto de mão e/ou velocidade de marcha. Além disso, observa-se na literatura que as definições operacionais de sarcopenia que incorporam medidas de função muscular produzem uma menor prevalência de sarcopenia em comparação com definições operacionais que empregam apenas massa muscular. A observação da baixa prevalência limitaria a oportunidade de identificação precoce e aplicação de estratégias de prevenção, para além de apresentar desafios no desenvolvimento de estratégias eficazes para reduzir a sarcopenia. Assim, acredita-se que a atual proposta e definição operacional não são definitivas e podem sofrer alterações consideráveis à medida que o conhecimento sobre a sarcopenia se desenvolve.

\section{AGRADECIMENTOS}

Vítor Oliveira agradece ao apoio recebido pela Capes durante o doutoramento (bolsista da Capes/Programa de Doutorado Sanduíche no Exterior/Processo $\left.n^{\circ}\{88881.132132 / 2016-01\}\right)$, período em que desenvolveu a sua tese intitulada Sarcopenia em pessoas vivendo com o vírus da imunodeficiência humana.

\section{REFERÊNCIAS BIBLIOGRÁFICAS}

1. Rosenberg IH. Summary comments. Am J Clin Nutr. 1989;50(5):12313.

2. Rosenberg $\mathrm{IH}$. Sarcopenia: origins and clinical relevance. J Nutr. 1997;127(5 Suppl):990S-1S.

3. Cruz-Jentoft AJ, Baeyens JP, Bauer JM, Boirie Y, Cederholm T, Landi F, et al. Sarcopenia: European consensus on definition and diagnosis (report of the European Working Group on Sarcopenia in Older People). Age Ageing. 2010;39(4):412-23.

4. Anker SD, Morley JE, von Haehling S. Welcome to the ICD-10 code for sarcopenia. J Cachexia Sarcopenia Muscle. 2016;7(5):512-4.

5. Hardee JP, Lynch GS. Current pharmacotherapies for sarcopenia. Expert Opin Pharmacother. 2019;20(13):1645-57.

6. Baumgartner RN, Koehler KM, Gallagher D, Romero L, Heymsfield SB, Ross RR, et al. Epidemiology of sarcopenia among the elderly in New Mexico. Am J Epidemiol. 1998;147(8):755-63.

7. Cruz-Jentoft $A J$, Bahat $G$, Bauer J, Boirie $Y$, Bruyère $O$, Cederholm $T$, et al. Sarcopenia: revised European consensus on definition and diagnosis. Age Ageing. 2019;48(1):16-31.

8. Keller K, Engelhardt M. Strength and muscle mass loss with aging process: age and strength loss. Muscles Ligaments Tendons J. 2014;3(4): 346-50.

9. Kim TN, Park MS, Yang SJ, Yoo HJ, Kang HJ, Song W, et al. Prevalence and determinant factors of sarcopenia in patients with type 2 diabetes: the Korean Sarcopenic Obesity Study (KSOS). Diabetes Care. 2010;33(7):1497-9.

10. Chindapasirt J. Sarcopenia in cancer patients. Asian Pac J Cancer Prev. 2015;16(18):8075-7.

11. Wang T, Feng X, Zhou J, Gong H, Xia S, Wei Q, et al. Type 2 diabetes mellitus is associated with increased risks of sarcopenia and pre-sarcopenia in Chinese elderly. Sci Rep. 2016;6:38937.

12. Oliveira VH, Borsari AL, Webel AR, Erlandson KM, Deminice R. Sarcopenia in people living with the human immunodeficiency virus: a systematic review and meta-analysis. Eur J Clin Nutr. 2020;74(7):1009-21.

13. Shafiee G, Keshtkar A, Soltani A, Ahadi Z, Larijani B, Heshmat R. Prevalence of sarcopenia in the world: a systematic review and meta-analysis of general population studies. J Diabetes Metab Disord. 2017;16:21.

14. Ethgen $O$, Beaudart $C$, Buckinx $F$, Bruyère $O$, Reginster JY. The future prevalence of sarcopenia in Europe: a claim for public health action. Calcif Tissue Int. 2017;100(3):229-34.

15. Du K, Goates S, Arensberg MB, Pereira S, Gaillard T. Prevalence of sarcopenia and sarcopenic obesity vary with race/ethnicity and advancing age. Divers Equal Health Care. 2018;15(4):175-83.

16. Diz JB, Leopoldino AA, Moreira BS, Henschke N, Dias RC, Pereira LS, et al. Prevalence of sarcopenia in older Brazilians: a systematic review and meta-analysis. Geriatr Gerontol Int. 2017;17(1):5-16.

17. Dodds RM, Granic A, Davies K, Kirkwood TB, Jagger C, Sayer AA. Prevalence and incidence of sarcopenia in the very old: findings from the Newcastle 85+ Study. J Cachexia Sarcopenia Muscle. 2017;8(2):229-37.

18. Yazar T, Yazar HO. Prevalance of sarcopenia according to decade. Clin Nutr ESPEN. 2019;29:137-41.

19. Ngeuleu A, Allali F, Medrare L, Madhi A, Rkain H, Hajjaj-Hassouni N. Sarcopenia in rheumatoid arthritis: prevalence, influence of disease activity and associated factors. Rheumatol Int. 2017;37(6):1015-20.

20. Kim G, Kang SH, Kim MY, Baik SK. Prognostic value of sarcopenia in patients with liver cirrhosis: a systematic review and meta-analysis. PLoS One. 2017;12(10):e0186990.

21. Murata Y, Kadoya Y, Yamada S, Sanke T. Sarcopenia in elderly patients with type 2 diabetes mellitus: prevalence and related clinical factors. Diabetol Int. 2017;9(2):136-42.

22. Addison O, Prior SJ, Kundi R, Serra MC, Katzel LI, Gardner AW, et al. Sar- 
copenia in peripheral arterial disease: prevalence and effect on functional status. Arch Phys Med Rehabil. 2018;99(4):623-8.

23. Ryan E, McNicholas D, Creavin B, Kelly ME, Walsh T, Beddy D. Sarcopenia and inflammatory bowel disease: a systematic review. Inflamm Bowel Dis. 2019;25(1):67-73.

24. Jones SE, Maddocks M, Kon SS, Canavan JL, Nolan CM, Clark AL, et al. Sarcopenia in COPD: prevalence, clinical correlates and response to pulmonary rehabilitation. Thorax. 2015;70(3):213-8.

25. Pereira RA, Cordeiro AC, Avesani CM, Carrero JJ, Lindholm B, Amparo $F C$, et al. Sarcopenia in chronic kidney disease on conservative therapy: prevalence and association with mortality. Nephrol Dial Transplant. 2015;30(10):1718-25.

26. Souza VA, Oliveira D, Barbosa SR, Corrêa JO, Colugnati FA, Mansur HN, et al. Sarcopenia in patients with chronic kidney disease not yet on dialysis: analysis of the prevalence and associated factors. PLoS One. 2017;12(4):e0176230.

27. Onishi S, Shiraki M, Nishimura K, Hanai T, Moriwaki H, Shimizu M. Prevalence of sarcopenia and its relationship with nutritional state and quality of life in patients with digestive diseases. J Nutr Sci Vitaminol. 2018;64(6):445-53.

28. Corallo C, Fioravanti A, Tenti S, Pecetti G, Nuti R, Giordano N. Sarcopenia in systemic sclerosis: the impact of nutritional, clinical, and laboratory features. Rheumatol Int. 2019;39(10):1767-75.

29. Hao Q, Hu X, Xie L, Chen J, Jiang J, Dong B, et al. Prevalence of sarcopenia and associated factors in hospitalised older patients: a cross-sectional study. Australas J Ageing. 2018;37(1):62-7.

30. Vetrano DL, Pisciotta MS, Laudisio A, Lo Monaco MR, Onder G, Brandi $\checkmark$, et al. Sarcopenia in Parkinson disease: comparison of different criteria and association with disease severity. J Am Med Dir Assoc. 2018;19 (6):523-7.

31. Liu P, Hao Q, Hai S, Wang H, Cao L, Dong B. Sarcopenia as a predictor of all-cause mortality among community-dwelling older people: a systematic review and meta-analysis. Maturitas. 2017;103:16-22.

32. Hu X, Zhang L, Wang H, Hao Q, Dong B, Yang M. Malnutrition-sarcopenia syndrome predicts mortality in hospitalized older patients. Sci Rep. 2017;7(1):3171.

33. Villaseñor A, Ballard-Barbash R, Baumgartner K, Baumgartner R, Berstein L, McTiernan A, et al. Prevalence and prognostic effect of sarcopenia in breast cancer survivors: the HEAL Study. J Cancer Surviv. 2012; 6(4):398-406.

34. Sousa AS, Guerra RS, Fonseca I, Pichel F, Ferreira S, Amaral TF. Financial impact of sarcopenia on hospitalization costs. Eur J Clin Nutr. 2016; 70(9):1046-51.

35. Janssen I, Shepard DS, Katzmarzyk PT, Roubenoff R. The healthcare costs of sarcopenia in the United States. J Am Geriatr Soc. 2004;52(1): 80-5.

36. Steffl M, Sima J, Shiells K, Holmerova I. The increase in health care costs associated with muscle weakness in older people without long-term illnesses in the Czech Republic: results from the Survey of Health, Ageing and Retirement in Europe (SHARE). Clin Interv Aging. 2017;12:2003-7.

37. Dawson-Hughes $B$, Bischoff-Ferrari $H$. Considerations concerning the definition of sarcopenia. Osteoporos Int. 2016;27(11):3139-44.

38. Janssen I, Baumgartner RN, Ross R, Rosenberg IH, Roubenoff R. Skeletal muscle cutpoints associated with elevated physical disability risk in older men and women. Am J Epidemiol. 2004;159(4):413-21.

39. Muscaritoli M,Anker SD,Argilés J,Aversa Z, Bauer JM, Biolo G, et al. Consensus definition of sarcopenia, cachexia and pre-cachexia: joint document elaborated by Special Interest Groups (SIG) "cachexia-anorexia in chronic wasting diseases" and "nutrition in geriatrics". Clin Nutr. 2010;29(2):154-9.

40. Fielding RA, Vellas B, Evans WJ, Bhasin S, Morley JE, Newman AB, et al. Sarcopenia: an undiagnosed condition in older adults. Current consensus definition: prevalence, etiology, and consequences. J Am Med Dir Assoc. 2011;12(4):249-56.

41. Schaap LA, Koster A, Visser M. Adiposity, muscle mass, and muscle strength in relation to functional decline in older persons. Epidemiol Rev. 2013;35:51-65.

42. Leong DP, Teo KK, Rangarajan S, Lopez-Jaramillo P, Avezum Jr A, Orlan$\operatorname{dini} A$, et al. Prognostic value of grip strength: findings from the Prospective Urban Rural Epidemiology (PURE) study. Lancet. 2015;386 (9990):266-73.

43. Menant JC, Weber F, Lo J, Sturnieks DL, Close JC, Sachdev PS, et al. Strength measures are better than muscle mass measures in predicting health-related outcomes in older people: time to abandon the term sarcopenia? Osteoporos Int. 2017;28(1):59-70.

44. Malmstrom TK, Miller DK, Simonsick EM, Ferrucci L, Morley JE. SARCF: a symptom score to predict persons with sarcopenia at risk for poor functional outcomes. J Cachexia Sarcopenia Muscle. 2016;7(1):28-36.

45. Barbosa-Silva TG, Menezes AM, Bielemann RM, Malmstrom TK, Gonzalez MC. Enhancing SARC-F: improving sarcopenia screening in the clinical practice. J Am Med Dir Assoc. 2016;17(12):1136-41.

46. Roberts HC, Denison HJ, Martin HJ, Patel HP, Syddall H, Cooper C, et al. A review of the measurement of grip strength in clinical and epidemiological studies: towards a standardised approach. Age Ageing. 2011;40(4):423-9.

47. Sousa-Santos AR, Amaral TF. Differences in handgrip strength protocols to identify sarcopenia and frailty: a systematic review. BMC Geriatr. 2017;17(1):238.

48. MacDermid J, Solomon G, Fedorczyk J, Valdes K. Clinical assessment recommendations: impairment-based conditions. 3rd ed. Mount Laurel: American Society of Hand Therapists; 2015.

49. Nakano MM. Versão brasileira da Short Physical Performance Battery (SPPB): adaptação cultural e estudo da confiabilidade [dissertation]. Campinas: Unicamp; 2007.

50. Phu S, Vogrin S, Zanker J, Hassan EB, Al Saedi A, Duque G. Agreement between initial and revised European working group on sarcopenia in older people definitions. J Am Med Dir Assoc. 2019;20(3):382-3.e1.

51. Rothney MP, Brychta RJ, Schaefer EV, Chen KY, Skarulis MC. Body composition measured by dual-energy $\mathrm{X}$-ray absorptiometry half-body scans in obese adults. Obesity. 2009;17(6):1281-6.

52. Kyle UG, Genton L, Hans D, Pichard C. Validation of a bioelectrical impedance analysis equation to predict appendicular skeletal muscle mass (ASMM). Clin Nutr. 2003;22(6):537-43.

53. Lukaski HC, BolonchukWW, Hall CB, Siders WA. Validation of tetrapolar bioelectrical impedance method to assess human body composition. J Appl Physiol. 1986;60(4):1327-32.

54. Guralnik JM, Simonsick EM, Ferrucci L, Glynn RJ, Berkman LF, Blazer DG, et al. A short physical performance battery assessing lower extremity 
function: association with self-reported disability and prediction of mortality and nursing home admission. J Gerontol. 1994;49(2): M85-94.

55. Reiss J, Iglseder B, Alzner R, Mayr-Pirker B, Pirich C, Kässmann H, et al. Consequences of applying the new EWGSOP2 guideline instead of the former EWGSOP guideline for sarcopenia case finding in older patients. Age Ageing. 2019;48(5):719-24.

\section{CONFLITO DE INTERESSES}

Os autores declaram não possuir quaisquer conflitos de interesse.

\section{FONTES DE FINANCIAMENTO}

Este estudo não recebeu qualquer financiamento.

\author{
ENDEREÇO PARA CORRESPONDÊNCIA \\ Vitor Oliveira \\ E-mail: vitorhfo@hotmail.com \\ https://orcid.org/0000-0002-2273-1408
}

Recebido em 01-07-2020

Aceite para publicação em 26-06-2021

\section{ABSTRACT}

\section{UPDATE ON THE DEFINITION, CONSEQUENCES, AND DIAGNOSIS OF SARCOPENIA: A LITERATURE REVIEW}

Introduction: Sarcopenia is defined as a progressive and generalized skeletal muscle disorder that is associated with a greater likelihood of health complications, including falls, fractures, physical disability, and mortality. Sarcopenia, which was previously described as a condition inherent to age, has different recognized causal factors and affects many individuals regardless of age. Due to its importance and consequences, sarcopenia is currently recognized as a pathological condition and has a code in the international classification of diseases (ICD $10-$ M62.84).

Purpose: To present a literature review on the causal factors, epidemiology, and methods of diagnosis of sarcopenia currently employed.

Data sources: MEDLINE/PubMed, Embase, and SciELO (initial research) and references of selected papers from the primary research.

Review methods: A combination of the following terms was used: sarcopenia, diagnosis, operational definition, prevalence, consequences. Original papers, review papers, clinical guidelines, and consensus in English and Portuguese published between 1989 (the year of the proposition of the term sarcopenia) until May 2020 were considered.

Results: The main references selected were papers related to the sarcopenia definition. We also selected papers related to tests and assessments used for the diagnosis of sarcopenia, and papers describing the prevalence of sarcopenia in different populations.

Conclusions: Considering the historical evolution of sarcopenia diagnosis, it is notorious the attempt to bring scientific knowledge closer to clinical practice with the suggestion of methods and cutoff points that facilitate the immediate identification of sarcopenia. However, there are many different propositions and operational definitions of sarcopenia making it difficult to determine its actual prevalence. Despite recent developments in the understanding of sarcopenia, a definitive operational definition and the best way to diagnose sarcopenia are still a matter of debate. Thus, we believe that the current operational definition is not definitive and can undergo considerable changes as knowledge about this field advances.

Keywords: Sarcopenia; Frailty; Aging; Epidemiology. 